\title{
Monocyte-derived chicken macrophages exposed to Eimeria tenella sporozoites display reduced susceptibility to invasion by Toxoplasma gondii tachyzoites
}

Runhui Zhang ( $\nabla$ zhangrunhui423@gmail.com )

Institue of Parasitology, Leipzig University

Wanpeng Zheng

Institute of parasitology, Universitat Leipzig

Arwid Daugschies

Institute of parasitology, University Leipzig

Berit Bangoura

Department of Veterinary Sciences, University of Wyoming

Short report

Keywords: Toxoplasma gondii, Eimeria tenella, co-infection, chicken macrophages, live cell imaging

Posted Date: April 13th, 2020

DOI: https://doi.org/10.21203/rs.3.rs-21822/v1

License: (c) (1) This work is licensed under a Creative Commons Attribution 4.0 International License.

Read Full License 


\section{Abstract}

Background: Both Eimeria (E.) tenella and Toxoplasma (T.) gondii are common apicomplexan parasites in chickens. Host cell invasion by both protozoans includes gliding motility, host cell attachment and active penetration. Chicken macrophages as phagocytic cells participate in the innate host immune response against these two parasites.

Methods: In this study, primary chicken monocyte-derived macrophages (MMs) were infected with both pathogens to investigate mutual and host-parasite interactions. MMs cultures were assigned to groups that were infected with E. tenella, T. gondii, or both. In co-infected cultures, MMs were first exposed to $E$. tenella sporozoites for 2 hours. Afterwards, T. gondii tachyzoite infection was performed. Live-cell imaging was carried out to observe cell invasion and survival of $T$. gondii by single parasite tracking over a period of 20 hours post infection (hpi). Quantitative analysis for parasite replication was performed at $2,6,12$ and 24 hpi by real-time PCR (qPCR).

Results: We found that during early co-infection T. gondii tachyzoites adhered for more than 4 hours to macrophages. Although they displayed high motility, ability to penetrate the cell membrane of the potential host cell was reduced. qPCR results confirmed that significantly less $T$. gondii entered $E$. tenellaactivated MMs at $2 \mathrm{hpi}$, and a reduced proportion of intracellular T. gondii survived and replicated in these cells at $24 \mathrm{hpi}$.

Conclusions: We conclude that E. tenella modulates host cell responses to another apicomplexan agent, T. gondii, reducing active invasion and multiplication in chicken primary macrophages.

\section{Background}

Apicomplexan protozoa are obligate intracellular parasites causing a variety of diseases in animals and humans. Toxoplasma (T.) gondii infects almost all euthermic animal species and may also infect humans. About one third of the global human population are supposed to be infected by this zoonotic pathogen [1]. Chickens are considered to be an important reservoir of $T$. gondii. Especially free-ranging and back-yard chickens have been reported to show high seroprevalence rates for T. gondii [2]. On the other hand, coccidiosis caused by Eimeria species is a widely distributed major parasitic disease of poultry, one of the most pathogenic species in chickens being $E$. tenella.

Upon adherence to the host cell membrane apicomplexan parasites start to penetrate into the host cell by forming a so-called moving junction initiated by organelles of the apical complex at the anterior end of the parasites [3]. This moving junction supports the establishment of the parasitophorous vacuole (PV) separating the parasite from the host cell cytoplasma. The adhesive proteins produced by micronemes located in the apical region of the invasive parasite stages allow gliding motility which is essential for the active invasion by $T$. gondii [4]. In chickens, macrophages play a crucial role in identification and phagocytosis of pathogens including protozoan thus serving as a first line of innate immune defence [5]. However, T. gondii tachyzoites are capable to actively invade macrophages which occurs even faster than 
phagocytosis displayed by a macrophage [6]. T. gondii tachyzoites are able to replicate in chicken blood monocyte-derived macrophages [7]. In experimental infection of $E$. tenella in naïve chicken, sporozoites were situated mainly within or next to the lamina propria that was infiltrated with macrophages in response to the infection [8]. Sporozoite-bearing macrophages are able to transport Eimeria sporozoites to the proper site of the intestinal mucosa [9]. However, E. tenella survival and development is rather poor in cultured chicken macrophages [10].

Concomitant infections by protozoan parasites and other microorganisms attract increasing attention in animals and humans both under natural conditions and in experimental in vivo and in vitro studies. For instance, probiotic bacteria reduced oocyst excretion of E. acervulina in chicken [11] as well as in vitro invasion of E. tenella into Madin-Darby bovine kidney cells [12]. Host immune responses and consequently clinical signs of disease are modulated in co-infected animals. In general, the immune response is dominated by Th1 during protozoan infection whereas Th2 response is typical for helminth infection [13]. Regarding interaction during co-infection by protozoa, it was reported that no competitive effects exist in mixed Eimeria species infection [14]. However, T. gondii replication was increased during co-infection with Trypanosoma in rats [15]. On the other hand, T. gondii supports Plasmodium replication in a rat model [16]. Natural co-occurrence of $T$. gondii and Eimeria in the same host has been reported. For example, both parasites were found concurrently in blood and organs of a heavily diseased sparrow [17]. Furthermore, wild rabbits tested seropositive for both $T$. gondii and Eimeria stiedae infection in Scotland [18].

In spite of high seroprevalence rates reported for both $T$. gondii and E. tenella in chickens, little is known to date about the mutual interplay between these two parasites, particularly during host cell invasion. Experimental in vivo and ex vivo co-infection models in chicken macrophages were established recently and thus suitable tools are now available for further co-infection studies [19, 20]. Results demonstrated that the mutual interaction during co-infection modulated both parasite replication as well as the host immune response. In our recent study, macrophage phagocytosis was distinctly altered during in vitro coinfection by $T$. gondii and $E$. tenella (unpublished data).

In the current study, we used live-cell imaging of $T$. gondii and/or E. tenella infected cells to monitor parasite invasion and survival at the single cell-parasite level in both mono-infected and co-infected cultures. The study focuses on the period of early parasite invasion in monocyte-derived chicken macrophages. A previous study showed that asexual stages of T. gondii and Eimeria displayed ultrastructural similarities [21]. Moreover, host cell invasion mechanisms, micronemal adhesins, gliding motility of both $T$. gondii tachyzoites and Eimeria sporozoites were identified to be affected in vitro by the same inhibitor of protozoan cGMP-dependent protein kinase [22]. Thus, we are particularly interested in whether the considerable capacity of $T$. gondii to invade and survive in macrophages is affected by hostspecific $E$. tenella infection. Parasite tracking was performed to determine the motility of $T$. gondii during the invasion phase following co-infection by E. tenella. The life span of individual T. gondii was also monitored by live cell imaging. 


\section{Methods}

\section{Parasites and host cells}

Tachyzoites of $T$. gondii RH - green fluorescent protein (GFP) strain (kindly provided by Professor Dominique Soldati-Favre, University of Geneva Medical School, Switzerland) were maintained at $37^{\circ} \mathrm{C}$ with $5 \% \mathrm{CO}_{2}$ in human foreskin fibroblast (HFF) cells. Free tachyzoites were collected from the culture medium for infection. The sporozoites of $E$. tenella Houghton - yellow fluorescent protein (YFP) strain (kindly provided by Prof. Dr. Suo, China Agricultural University, China) were obtained from oocysts by excystation following an established protocol [23].

The animal experiments performed to collect chicken blood samples were approved by the responsible authorities (Landesdirektion Sachsen, Germany, trial registration number V13/10). Chicken peripheral blood mononuclear cells (PBMCs) were isolated from heparinized whole blood of adult chickens according to the established protocols with slight modifications kindly provided by Dr. Braukmann, Friedrich-Loeffler-Institute Jena, Germany. Briefly, PBMCs were separated from $2 \mathrm{ml}$ blood by centrifugation ( $250 \times$ g, $45 \mathrm{~min}$ ) with Biocoll (density $1.077 \mathrm{~g} / \mathrm{ml}$; Biochrom AG, Berlin, Germany). Isolated PBMCs were resuspended and washed in $5 \mathrm{~mL}$ PBS (centrifugation $350 \times \mathrm{g}, 30 \mathrm{~min}$ ) following prewarmed $5 \mathrm{~mL}$ RPMI-1640 medium (Sigma, Taufkirchen, Germany) (centrifugation $350 \times$ g, $20 \mathrm{~min}$ ). Afterwards, $5 \times 10^{6}$ PBMCs/well were resuspended in 24-well-plates in RPMI-1640 with 5\% chicken serum and $5 \%$ fetal bovine serum, penicillin $(100 \mathrm{U} / \mathrm{mL}, \mathrm{PAA})$, streptomycin $(0.1 \mathrm{mg} / \mathrm{mL}, \mathrm{PAA})$, and amphotericin $\mathrm{B}(0.0025 \mathrm{mg} / \mathrm{mL}, \mathrm{PAA})$. After $72 \mathrm{~h}$ incubation $\left(41^{\circ} \mathrm{C}, 5 \% \mathrm{CO}_{2}\right)$ PMBCs were trypsinized by Biotase ${ }^{\circledR}$ (Biochrom, Berlin, Germany) at $37^{\circ} \mathrm{C}$ for $30 \mathrm{~min}$. Detached monocyte devived macrophages (MMs) were counted under the microscope. $10^{3} \mathrm{MMs}$ were seeded in the microscope imaging chamber (micro-insert 4 well, Ibidi, Martinsried, Germany) for $24 \mathrm{~h}\left(41^{\circ} \mathrm{C}, 5 \% \mathrm{CO}_{2}\right)$ for live cell imaging. For parasite quantification by qPCR, approximately $10^{5} \mathrm{MMs}$ were seeded to 24 -well-plates for $24 \mathrm{~h}\left(41^{\circ} \mathrm{C}, 5 \% \mathrm{CO}_{2}\right)$. The MMs were purified by rinsing off non-adherent cells once at $24 \mathrm{~h}$ and twice before infection.

\section{Infection}

Two experiments were conducted in this study (Fig. 1): Experiment 1 was designed to visualize parasite invasion; Experiment 2 was performed with increased MMs population at the same infection ratio to quantify parasite stages by qPCR for the different infection groups.

Study design for experiment 1 (Fig. 1A):

For imaging, cell cultures were assigned to four groups ( $n=2$ per group). In co-infected group $\mathrm{Cl}, \mathrm{MMs}$ were exposed to $2 \times 10^{3}$ E. tenella sporozoites $2 \mathrm{~h}$ before $T$. gondii infection ( $-2 \mathrm{hpi}$ ). Group LPS cultures served as positive controls and were stimulated at the same time with $1 \mu \mathrm{g} / \mathrm{mL}$ lipopolysaccharide (LPS). Cultures of group $\mathrm{Tg}$ (monoinfection with T. gondii) and group NC (negative control) were not exposed to 
LPS stimulation. At 0 hpi cultures of groups $\mathrm{Cl}$, LPS, and Tg were infected with $2 \times 10^{3} \mathrm{~T}$. gondii tachyzoites per well. Group NC remained uninfected. All cultures were observed until $20 \mathrm{hpi}$. The whole experiment was repeated once biologically.

Study design for experiment 2 (Fig. 1B):

For quantification by qPCR, cell cultures kept in 24-well plates were assigned to five groups ( $n=5$ cultures/group). At $-2 \mathrm{hpi}$, MMs of group $\mathrm{Cl}$ (co-infection) and group $\mathrm{Et}$ (single infection with E. tenella) were exposed to $2 \times 10^{5}$ E. tenella sporozoites per culture. Group LPS cultures were pretreated with $1 \mu \mathrm{g} / \mathrm{ml}$ LPS at the same time. Group Tg was infected with $2 \times 10^{5} \mathrm{~T}$. gondii tachyzoites per well at 0 hpi while group NC served as untreated, uninfected negative control. The cultures were maintained until 24 hpi.

\section{Live-cell imaging of $T$. gondii in MMs}

DRAQ7 dye was used to assess the viability of parasites and macrophages in each group. Prior to imaging, $3 \mu \mathrm{L}$ DRAQ7 dye (Biostatus, Leicestershire, UK), a nuclear stain selective for dead cells, was added to all cell cultures immediately after exposure to E. tenella or LPS treatment (-2 hpi). The viability of parasites and cells were controlled by fluorescent microscopy prior to T. gondii infection at 0 hpi. Cells were viewed for fluorescence by confocal laser scanning microscope (CLSM) (TCS-SP8, Leica, Bensheim, Germany) using 4 channels at $488 \mathrm{~nm}, 514 \mathrm{~nm}, 633 \mathrm{~nm}$ laser line and wide field. Basic imaging parameters were $40 \times$ objective, $10 \times$ ocular (NA 0.90), $1024 \times 1024$ dpi, 6 Z-stacks $(4 \mu \mathrm{m})$. To avoid crosstalk between channels, images were collected in line sequential mode.

Incubation conditions $\left(41^{\circ} \mathrm{C}, 5 \% \mathrm{CO}_{2}\right.$ and $99 \%$ humidity) were controlled using an incubation chamber (Tokai-Hit, Shizuoka, Japan) over the whole observation period. The timeframe of infection and imaging is shown in Fig. 1A. Briefly, after adding DRAQ7, approximately 20 random fields of cells were selected and captured according to groups Cl, LPS, and Tg from 2 individual wells per group. Image collection for time-lapse imaging of tachyzoite motility was performed at an interval of 10 min per frame until $2 \mathrm{hpi}$. Image acquisition was interrupted from $2 \mathrm{hpi}$ to $3 \mathrm{hpi}$ to add 20 additional fields per group which contained intracellular T. gondii in group $\mathrm{Cl}$ and LPS. All fields were observed for further $4 \mathrm{~h}$ until $7 \mathrm{hpi}$ at an interval of 30 min per frame. Subsequently, at least 100 cells with live intracellular T. gondii (1-2 tachyzoites / cell) were collected and captured at $7 \mathrm{hpi}$ and 20 hpi by CLSM for groups $\mathrm{Cl}$ and $\mathrm{Tg}$. For group LPS, only $71 \mathrm{MMs}$ with live intracellular $T$. gondii were selected due to a generally low number of $T$. gondii-positive cells observed in this group. Six fields were analysed randomly for group NC in parallel.

Images of stacks were obtained using LAS X software (Leica, Bensheim, Germany). Stacks were analysed with Imaris $\AA^{\circledR}$ software version 9.3 (Bitplane, Abingdon, UK) using functions of spot detection and tracking parasite motility and viability. 


\section{Parasite Quantification By Quantitative Real-time Pcr (qpcr)}

For all infection groups (Fig. 1B), complete cell populations from a subset of cell culture wells were collected at 2, 6, 12, and $24 \mathrm{hpi}$ and additionally at $0 \mathrm{hpi}$ in group Et. DNA was extracted using the QIAamp DNA Mini Kit ${ }^{\circledR}$ (Qiagen, Hilden, Germany) following the manufacturer's instructions. qPCR was performed using the CFX Connect Real-Time PCR Detection System (Bio-Rad, Hercules, USA). Data represent the mean of three replicates with an acceptable standard deviation of less than $0.5 \mathrm{for} C \mathrm{Ct}$ values.

T. gondii multiplication was analyzed by a probe-based qPCR detecting the 529-bp repeat element [24]. Standard curve was developed by data obtained for gradient 10 -fold dilutions of initially $10^{7}$ tachyzoites. qPCR was conducted in a total volume of $25 \mu \mathrm{L}: 5 \mu \mathrm{L}$ of sample DNA, $12.5 \mu \mathrm{L}$ of Master Mix, 3.2 $\mu \mathrm{L}$ of DNase/RNase free water (Gibco ${ }^{\text {TM }}$, Life Technologies, USA), $2.5 \mu \mathrm{L}$ of $2 \mu \mathrm{M}$ TaqMan probe and $0.9 \mu \mathrm{L}$ of each $25 \mu \mathrm{M}$ forward and reverse primer (5'-CACAGAAGGGACAGAAGT and 5'-TCGCCTTCATCTACAGTC$\left.3^{\prime}\right)$. The cycling program consisted of $95^{\circ} \mathrm{C}$ for $15 \mathrm{~min}$, followed by 40 cycles of $95^{\circ} \mathrm{C}$ for $15 \mathrm{~s}, 60^{\circ} \mathrm{C}$ for $1 \mathrm{~min}$, and $72^{\circ} \mathrm{C}$ for $15 \mathrm{~s}$.

ITS1 fragment quantification was used to assess the replication of E. tenella by a SYBR Green-based PCR [25]. The relative copy number of E. tenella DNA was implemented by measurement of pSCA-17 plasmid standard dilution as described before [26]. qPCR was conducted in a total volume of $20 \mu \mathrm{L}: 2 \mu \mathrm{L}$ of sample DNA eluate, $10 \mu \mathrm{L}$ of SYBR Green master mix (Thermo Fisher Scientific, Darmstadt, Germany), $7.2 \mu \mathrm{L}$ of water, $0.9 \mu \mathrm{L}$ of $25 \mu \mathrm{M}$ forward and reverse primer (5'-aacctgactgtgcaagcatc-3' and 5'atcatagacagccgtgccag- $3^{\prime}$ ). The cycling program consisted of heating to $95^{\circ} \mathrm{C}$ for $5 \mathrm{~min}$, followed by 40 cycles at $95^{\circ} \mathrm{C}$ for $30 \mathrm{~s}, 55^{\circ} \mathrm{C}$ for $20 \mathrm{~s}$, and $72^{\circ} \mathrm{C}$ for $20 \mathrm{~s}$. A subsequent melting curve analysis $\left(95^{\circ} \mathrm{C}\right.$ for $1 \mathrm{~min}, 55^{\circ} \mathrm{C}$ for $30 \mathrm{~s}, 0.5^{\circ} \mathrm{C} / \mathrm{s}$ ) was performed to create the dissociation curve.

\section{Statistical analysis}

Statistical analysis was performed by using Imaris ${ }^{\circledR}$ and GraphPad Prism ${ }^{\circledR}$ (version 8, San Diego, CA, USA) software. Kolmogorov-Smirnov test was performed to test for normal distribution. Statistical significance was assessed by two-way ANOVA for data with normal distribution and Tukey's multiple comparisons test for values that did not follow normal distribution.

\section{Results}

\section{Live cell imaging of T. gondii in MMs}

In general, penetration of $T$. gondii tachyzoites into the MMs started within $2 \mathrm{hpi}$ after tachyzoites were seeded into cultures. CLSM analysis (Fig. 2A) of group Cl cultures showed that $T$. gondii tachyzoites remained loosely adherent to MMs for more than 4 hours in most cases before they started to actively invade the host cell. During this phase, distinct helical gliding motility of tachyzoites was seen. This 
untypical behavior of tachyzoites in group $\mathrm{Cl}$ was observed regardless whether intracellular E. tenella sporozoites were still alive or dead. An additional movie file shows this in more detail (see Additional file 1). In contrast, tachyzoites, which were actively or passively incorporated into MMs after attachment, were seen within minutes in the mono-infected group $\mathrm{Tg}$ and the LPS-treated group.

Tachyzoites showed lowest motility in group LPS at most of the captured time points (Fig. 2B). In group $\mathrm{Cl}$, significantly higher motility of tachyzoites were observed than in group LPS over the period from $10 \mathrm{~min}$ to $120 \mathrm{~min}$ post infection $(\mathrm{p}<0.05)$; with the exception of 70 to $110 \mathrm{~min}$. Comparing between groups $\mathrm{Cl}$ and $\mathrm{Tg}$, tachyzoites of group $\mathrm{Cl}$ showed significantly higher motility $(\mathrm{p}<0.05)$ during the first 20 min. Average motility values in group $\mathrm{Cl}$ tended to remain higher than in both groups $\mathrm{Tg}$ and LPS with a significant difference $(p<0.05)$ at 80,90 and 110 min compared to group $\mathrm{Tg}$.

\section{Parasite Quantification}

In experiment 1, the relative intensity (RI) of green fluorescence of 27 to 30 individual intracellular $T$. gondii tachyzoites which were captured at 3 to $7 \mathrm{hpi}$ by CLSM were anlaysed in all infected groups. RI revealed no significant difference between groups $\mathrm{Tg}$ and $\mathrm{Cl}$ until $4 \mathrm{hpi}$. Thereafter, RI values remained on a consistently higher level in group $\mathrm{Cl}$ than in the monoinfected group $\mathrm{Tg}$ and in group LPS (Fig. 3A). The $\mathrm{RI}$ measured in group $\mathrm{Cl}$ displayed only slight variation from 3 to $7 \mathrm{hpi}$, whereas values started to decrease in group LPS and Tg following $4.5 \mathrm{hpi}$ or $5 \mathrm{hpi}$ with a statistically significant difference between groups $\mathrm{Cl}$ and LPS $(\mathrm{p}<0.05)$ at $4.5 \mathrm{hpi}$.

In general, less than $45 \%$ of traced intracellular tachyzoites of $T$. gondii were still alive or replicating in MMs at 20 hpi (Fig. 3B). At 20 hpi, the proportion of T. gondii-positive MMs was lower in group $\mathrm{Cl}(27 \%)$ than in group $\mathrm{Tg}(45 \%)$ and only slightly higher than in group LPS (21\%).

By qPCR, significantly lower DNA copy numbers $(\mathrm{p}<0.05)$ were determined at $2 \mathrm{hpi}$ for $T$. gondii in group $\mathrm{Cl}$ and group LPS compared to group Tg (Fig. 3C, left). The number of DNA copies remained on a low level in the two former groups until $12 \mathrm{hpi}$, followed by a steep increase until $24 \mathrm{hpi}$ in group $\mathrm{Cl}$ while values remained significantly lower in group LPS $(p<0.05)$. In group Tg, DNA copy numbers were higher than in the two other groups at $2 \mathrm{hpi}$, decreased steadily until $12 \mathrm{hpi}$ to a level similar to groups $\mathrm{Cl}$ and LPS and increased even more distinct than those recorded for group $\mathrm{Cl}$ thereafter.No statistical difference was observed between group $\mathrm{Cl}$ and $\mathrm{Tg}$ at this time point $(p>0.05)$. Likewise, no significant difference in DNA copy numbers was detected for $E$. tenella by comparison of groups $\mathrm{Et}$ and $\mathrm{Cl}$ over the entire observation period of $24 \mathrm{hpi}$ (Fig. 3C, right).

\section{Discussion}

After two hours of exposure to E. tenella sporozoites or LPS MMs generally maintained their viability which was demonstrated by negative staining for DRAQ7 (data not shown). A recent study by [20], confirmed earlier findings by [27] that $E$. tenella sporozoites were mostly located within chicken 
macrophages at $2 \mathrm{hpi}$. In the current study, most intracellular E. tenella sporozoites showed red fluorescescence after DRAQ7 staining instead of the expected yellow (YFP) fluorescence within 4 hpi to 6 hpi (data not shown), indicating their death. This CLSM finding corroborates the qPCR results demonstrate that the number of $E$. tenella declined to lowest numbers at 6 hpi (Fig. 3A, right). Co-infection with $T$. gondii did not exhibit a significant influence on $E$. tenella replication when compared to single $E$. tenella infection. Unfortunately, we could not quantify the $E$. tenella sporozoite numbers via YFP through time lapsing imaging because of a non-ideal YFP expression (about $80 \%$ ) in the available parasite strain.

Host cell invasion by $T$. gondii tachyzoites usually takes only 15 to $20 \mathrm{~s}[6], 1995)$. It is assumed that $T$. gondii tachyzoites and Eimeria sporozoites may invade and traverse several host cells by disrupting the host cell membrane [28]. In our time lapsing study, T. gondii tachyzoites were either floating free in the culture medium (unattached to macrophages) for hours or displayed a rapid entry into the macrophages, in both group LPS and group Tg. Interestingly, adherence of vital tachyzoites was prolonged for more than 4 hours in the co-infection cultures. It was previously demonstrated that most $T$. gondii tachyzoites remained adherent to murine macrophages that were treated with a phagocytosis inhibitor, Cytochalasin D [29]. Microneme exocytosis is necessary for host-cell entry of both T. gondii [30] and E. tenella [31]. The micronemes appear to be both structurally and functionally conserved between different members of the Apicomplexa [4]. We assumed that E. tenella infection may potentially hamper the entry of $T$. gondii into host cells by altering recognition of signal receptors or inhibiting phagocytosis.

Rapid invasion and egress are crucial to $T$. gondii survival and successful replication, thereby minimizing the exposure to destructive reaction by innate protection in a generally hostile extracellular environment [32]. Once inside the host cell, the parasite no longer moves [33]. The intracellular survival of T. gondii depends on the route by which the parasite enters the host cell. Although T. gondii tachyzoites are phagocytized and internalized through Fc receptor mediation [34], intracellular T. gondii can survive and replicate within their PV by blocking the host macrophage's pathways intended to initiate vacuolar acidification and parasite inactivation [35]. To quantify viable intracellular T. gondii, the intensity of GFP signal expression by the parasite was monitored in this study. It appeared from our observations according to RI values that $T$. gondii showed better tolerance and survival at the early stage of infection (until $7 \mathrm{hpi}$ ) if MMs were previously exposed to E. tenella (Fig. 3A).

Under the conditions of our experimental design (two T. gondii tachyzoites per cell), most infected host cells contained only one parasite at $7 \mathrm{hpi}$ in all infected groups. Compared to group LPS, By CLSM, only slightly high $T$. gondii-positive cells were monitored compared to group LPS. However, significant high number of total DNA copies of $T$. gondii was detected in group $\mathrm{Cl}$ compared to group LPS. Therefore, we conclude that the single cell observation by CLSM may not be completely comparable with quantification of DNA copies in cell cultures due to the different amount of cells considered. Nonetheless, results obtained with both methods indicated a reduced growth of $T$. gondii in chicken macrophages in a coinfection setting with $E$. tenella compared to mono-infection. 
Macrophages are not only professional phagocytes but also secrete cytokines in response to parasite infection. This innate immune response can be triggered in chicken macrophages by exposure to LPS [36]. A previous study showed that $T$. gondii blocked LPS-induced production of IL-12 and TNF-alpha in murine bone marrow derived macrophages [37]. For E. tenella, it is known that IL-1 $\beta$ and iNOS expression are significantly enhanced in chicken $\mathrm{HTC}$ macrophages by merozoites at $2 \mathrm{hpi}$ [38]. A recent in vitro study demonstrated down-regulation of IL-12 and iNOS in chicken macrophages during simultaneous coinfection by $T$. gondii and E. tenella [20]. From previous as well as the current data it appears that modulation of innate immunity in chicken differs during mono- and co-infection, which includes cytokine production and macrophage phagocytosis. However, data on concurrent infections with E. tenella and $T$. gondii are still scarce although both are considered to be common pathogens in poultry and thus deserve more attention.

\section{Conclusions}

In summary, life cell imaging by CLSM proved to be a useful tool to evaluate chicken macrophage invasion and / or phagocytosis during mono- and co-infection with two different apicomplexan parasites. It was demonstrated that the mechanisms of $T$. gondii invasion and survival appear to be altered in $E$. tenella-exposed macrophages. Further studies into macrophage signaling pathways, particularly modulation of the cytokine response, combined with image analysis and live cell imaging, will help to better understand the function and modulation of the innate immune response during apicomplexan invasion.

\section{Abbreviations}

PV: parasitophorous vacuole; GFP: green fluorescent protein; YFP: yellow fluorescent protein; PBMCs: peripheral blood mononuclear cells; LPS: lipopolysaccharide; CLSM: confocal laser scanning microscope;

\section{Declarations}

\section{Ethics approval and consent to participate}

The animal experiments performed to collect chicken blood samples were approved by the responsible authorities (Landesdirektion Sachsen, Germany, trial registration number V13/10)

\section{Consent for publication}

Not applicable

\section{Availability of data and materials}

Not applicable

Competing interests 
The authors declare that they have no competing interests

\section{Funding}

RZ received doctoral fellowships from Akademie für Tiergesundheit (AfT) and Karl-Enigk-Stiftung (KES), Germany. The authors acknowledge support from the German Research Foundation (DFG) and Leipzig University within the program of Open Access Publishing.

\section{Authors' Contributions}

RZ designed the study, wrote the manuscript, and performed the experiments and data analysis. WZ helped to perform cell imaging and manuscript drafting. AD and BB critically revised the study design, data interpretation and manuscript. All authors read and approved the final manuscript.

\section{Acknowledgments}

We thank Bioimaging Core Facility (BCF), Leipzig especially Dr. J. Kacza, and all members of the Institute of Parasitology, Leipzig University, for technical assistance and support. We are grateful to Mr. D. Hu for collecting samples.

\section{References}

1. Hill D, Dubey JP. Toxoplasma gondii: transmission, diagnosis and prevention. Clin Microbiol Infect. 2002;8:634-40.

2. Dubey JP. Toxoplasma gondii infections in chickens (Gallus domesticus): Prevalence, clinical disease, diagnosis and public health significance. Zoonoses Public Health. 2010;57:60-73.

3. Besteiro S, Dubremetz J-F, Lebrun M. The moving junction of apicomplexan parasites: a key structure for invasion. Cell Microbiol. 2011;13:797-805.

4. Sibley LD. How apicomplexan parasites move in and out of cells. Curr Opin Biotechnol. 2010;21:592-8.

5. Qureshi MA, Heggen CL, Hussain I. Avian macrophage: effector functions in health and disease. Dev Comp Immunol. 2000;24:103-19.

6. Morisaki JH, Heuser JE, Sibley LD. Invasion of Toxoplasma gondii occurs by active penetration of the host cell. J Cell Sci. 1995;108:2457-64.

7. Meirelles MN, Souza W de. Killing of Trypanosoma cruzi and Leishmania mexicana, and survival of Toxoplasma gondii, in chicken macrophages in vitro. J Submicrosc Cytol. 1985;17:327-34.

8. Vervelde L, Vermeulen AN, Jeurissen SHM. In situ characterization of leucocyte subpopulations after infection with Eimeria tenella in chickens. Parasite Immunol. 1996;18:247-56.

9. Trout JM, Lillehoi HS. Evidence of a role for intestinal CD8 + lymphocytes and macrophages in transport of Eimeria acervulina sporozoites. J Parasitol. 1993;79:790-2. 
10. Challey JR, Burns WC. The invasion of the cecal mucosa by Eimeria tenella sporozoites and their transport by macrophages. J Protozool. 1959;6:238-41.

11. Dalloul RA, Lillehoj HS, Shellem TA, Doerr JA. Enhanced mucosal immunity against Eimeria acervulina in broilers fed a Lactobacillus-based probiotic. Poult Sci. 2003;82:62-6.

12. Tierney J, Gowing H, van Sinderen D, Flynn S, Stanley L, McHardy N, et al. In vitro inhibition of Eimeria tenella invasion by indigenous chicken Lactobacillus species. Vet Parasitol. 2004;122:17182.

13. Marshall AJ, Brunet LR, van Gessel Y, Alcaraz A, Bliss SK, Pearce EJ, Denkers EY. Toxoplasma gondii and Schistosoma mansoni synergize to promote hepatocyte dysfunction associated with high levels of plasma TNF-a and early death in C57BL/6 mice. J Immunol. 1999;163:2089-97.

14. Cornelissen JBWJ, Swinkels WJC, Boersma WA, Rebel JMJ. Host response to simultaneous infections with Eimeria acervulina, maxima and tenella: A cumulation of single responses. Vet Parasitol. 2009;162:58-66.

15. Guerrero OM, Chinchilla M, Abrahams E. Increasing of Toxoplasma gondii (Coccidia, Sarcocystidae) infections by Trypanosoma lewisi (Kinetoplastida, Trypanosomatidae) in white rats. Rev Biol Trop. 1997:877-82.

16. Rifaat MA, Salem SA, Azab ME, el-Razik IA, Safer EH, Beshir SR, el-Shennawy SF. Experimental concomitant toxoplasma and malaria infection in rats. Folia parasitologica. 1984;31:97-104.

17. Manwell RD, Coulston F, Binckley EC, Jones VP. Mammalian and avian Toxoplasma. J Infect Dis. 1945;76:1-14.

18. Mason S, Dubey JP, Smith JE, Boag B. Toxoplasma gondii coinfection with diseases and parasites in wild rabbits in Scotland. Parasitology. 2015;142:1415-21.

19. Hiob L, Koethe M, Schares G, Goroll T, Daugschies A, Bangoura B. Experimental Toxoplasma gondii and Eimeria tenella co-infection in chickens. Parasitol Res. 2017:1-15.

20. Zhang R, thabet a, Hiob L, Zheng W, Daugschies A, Bangoura B. Mutual interactions of the apicomplexan parasites Toxoplasma gondii and Eimeria tenella with cultured poultry macrophages. Parasit Vectors. 2018;11:453.

21. Soares Medeiros LC, Gomes F, MACIEL LR, Seabra SH, Docampo R, Moreno S, et al. Volutin granules of Eimeria parasites are acidic compartments and have physiological and structural characteristics similar to acidocalcisomes. J Eukaryot Microbiol. 2011;58:416-23.

22. Wiersma HI, Galuska SE, Tomley FM, Sibley LD, Liberator PA, Donald RGK. A role for coccidian cGMPdependent protein kinase in motility and invasion. Int J Parasitol. 2004;34:369-80.

23. Rentería-Solís Z, Zhang R, Taha S, Daugschies A. A modified method for purification of Eimeria tenella sporozoites. Parasitol Res. 2020.

24. Edvinsson B, Lappalainen M, Evengård B. Real-time PCR targeting a 529-bp repeat element for diagnosis of toxoplasmosis. Clin Microbiol Infect. 2006;12:131-6. 
25. Kawahara F, Taira K, Nagai S, Onaga H, Onuma M, Nunoya T. Detection of five avian Eimeria species by species-specific real-time polymerase chain reaction assay. Avian Dis. 2008;52:652-6.

26. Thabet A, Alnassan AA, Daugschies A, Bangoura B. Combination of cell culture and qPCR to assess the efficacy of different anticoccidials on Eimeria tenella sporozoites. Parasitol Res. 2015;114:215563.

27. Long PL, Rose ME. Growth of Eimeria tenella in vitro in macrophages from chicken peritoneal exudates. Z Parasitenk. 1976;48:291-4. doi:.

28. Mota MM, Rodriguez A. Migration through host cells by apicomplexan parasites. Microbes Infect. 2001;3:1123-8.

29. Ryning FW, Remington JS. Effect of cytochalasin D on Toxoplasma gondii cell entry. Infect Immun. 1978;20:739-43.

30. Carruthers VB, Giddings OK, Sibley LD. Secretion of micronemal proteins is associated with Toxoplasma invasion of host cells. Cell Microbiol. 1999;1:225-35.

31. Bumstead J, Tomley F. Induction of secretion and surface capping of microneme proteins in Eimeria tenella. Mol Biochem Parasitol. 2000;110:311-21.

32. Hoff EF, Carruthers VB. Is Toxoplasma egress the first step in invasion? Trends Parasitol. 2002;18:251-5.

33. Dubremetz JF, Garcia-Réguet N, Conseil V, Fourmaux MN. Invited review Apical organelles and hostcell invasion by Apicomplexa. Int J Parasitol. 1998;28:1007-13.

34. Mordue DG, Sibley LD. Intracellular fate of vacuoles containing Toxoplasma gondii is determined at the time of formation and depends on the mechanism of entry. J Immunol. 1997;159:4452-9.

35. Sibley LD, Weidner E, Krahenbuhl JL. Phagosome acidification blocked by intracellular Toxoplasma gondii. Nature. 1985;315:416.

36. Hussain I, Qureshi MA. The expression and regulation of inducible nitric oxide synthase gene differ in macrophages from chickens of different genetic background. Vet Immunol Immunopathol. 1998;61:317-29.

37. Butcher BA, Denkers EY. Mechanism of entry determines the ability of Toxoplasma gondii to inhibit macrophage proinflammatory cytokine production. Infect Immun. 2002;70:5216-24.

38. Chow Y-P, Wan K-L, Blake DP, Tomley F, Nathan S. Immunogenic Eimeria tenella glycosylphosphatidylinositol-anchored surface antigens (SAGs) induce inflammatory responses in avian macrophages. PLoS One. 2011;6:e25233.

\section{Additional File 1}

Video microscopy of $T$. gondii invasion in a co-infected cell with an E. tenella sporozoite at 0-4.5 hpi. $T$. gondii is adherent on the cell which contains a vital E. tenella sporozoite. Yellow fluorescence: the nucleus of E. tenella sporozoites; green fluorescence: T. gondii tachyzoites; wide field: single macrophage. 


\section{(A) Experiment 1}

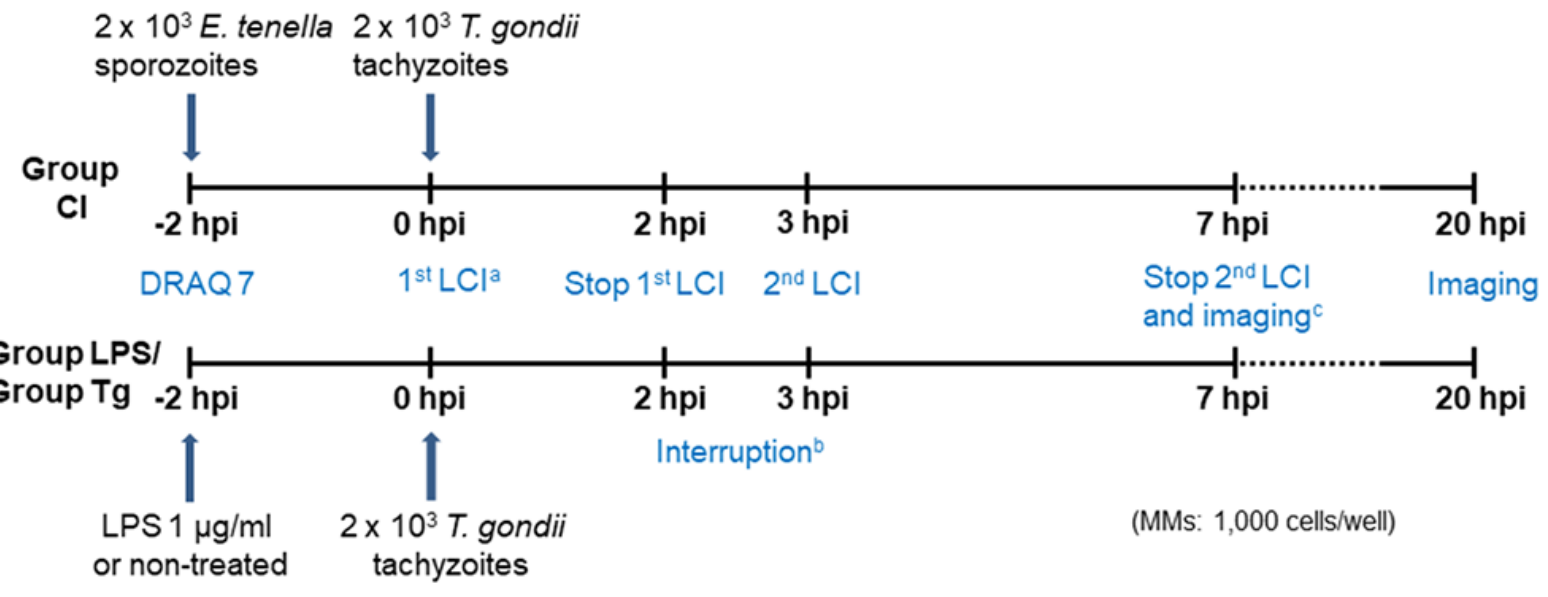

a Live cell imaging ( $\mathrm{LCl})$

b 1 hour Interruption to include new imaging fields of intracellular $T$. gondii

$\mathrm{C}^{\mathrm{s}}$ single cells with intracellular $T$. gondii were selected and captured by CLSM

(B) Experiment 2

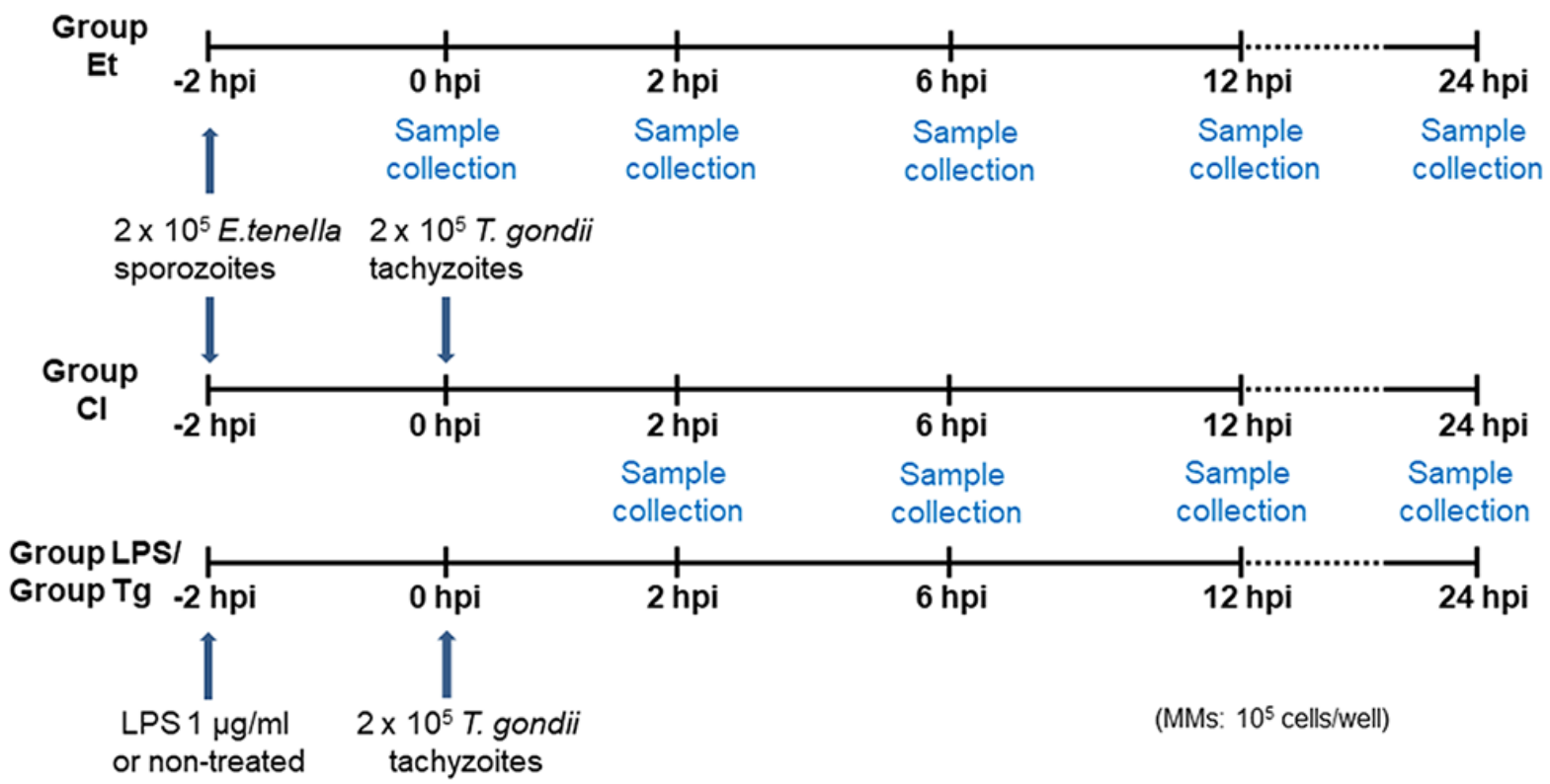

\section{Figure 1}

Schematic representation of infection and imaging. (A) Experiment 1 (live cell imaging). Images of group $\mathrm{NC}$ were collected at the same time points as in groups Cl, LPS and Tg. (B) Experiment 2 (parasite 
quantification by qPCR). Samples of group NC were collected at the same time point as in groups $\mathrm{Cl}$, LPS and $\mathrm{Tg}$.
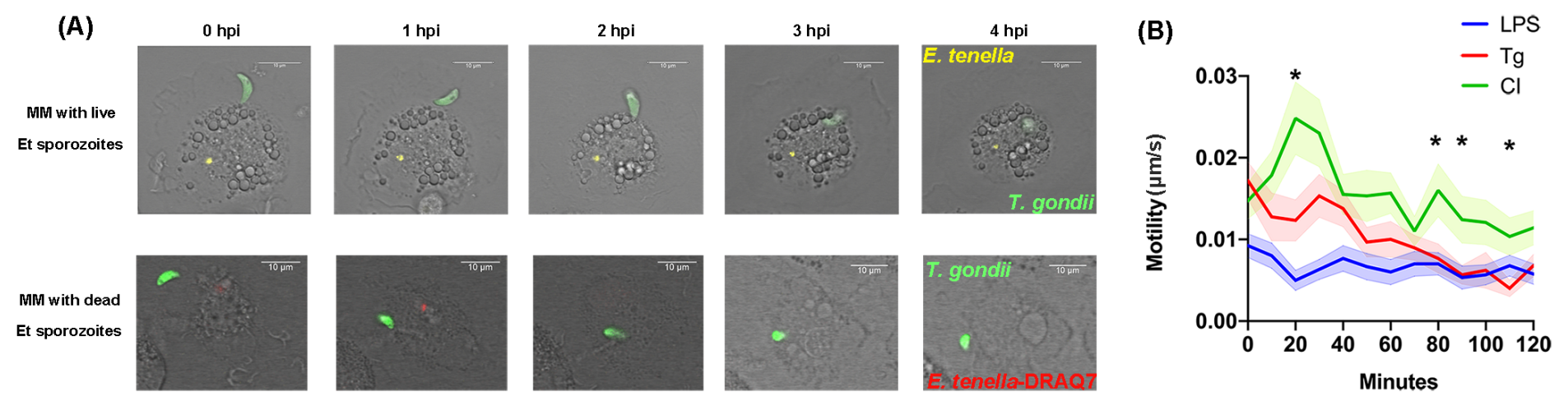

\section{Figure 2}

Invasion of T. gondii within $4 \mathrm{hpi}$. (A) Video microscopy of T. gondii invasion at 0-4 hpi in a cell previously exposed to E. tenella. (a) T. gondii is adherent on the cell which contains a vital E. tenella sporozoite. (b) T. gondii is adherent on the cell which contains a dead E. tenella sporozoite that was phagocytized and fused by macrophages at $2 \mathrm{hpi}$. Yellow fluorescence: the nucleus of E. tenella sporozoites; green fluorescence: T. gondii tachyzoites; wide field: single macrophage. (B) Motility (speed) of live T. gondii tachyzoites ( $0-120$ minutes). The motility of T. gondii tachyzoites $(n=30)$ was assessed by imaging every 10 minutes over 120 minutes. Each parasite was tracked over $70-100 \%$ of all time points excluding the occasional out-of-range movement or parasite death. Mean values per time point were calculated. LPS: LPS-treated, T. gondii mono-infection; Tg: T. gondii mono-infection; Cl: co-infection. ${ }^{*} \mathrm{p}<0.05, \mathrm{Cl}$ compared to Tg; Error bar: standard error of the mean (SEM) 
(A)

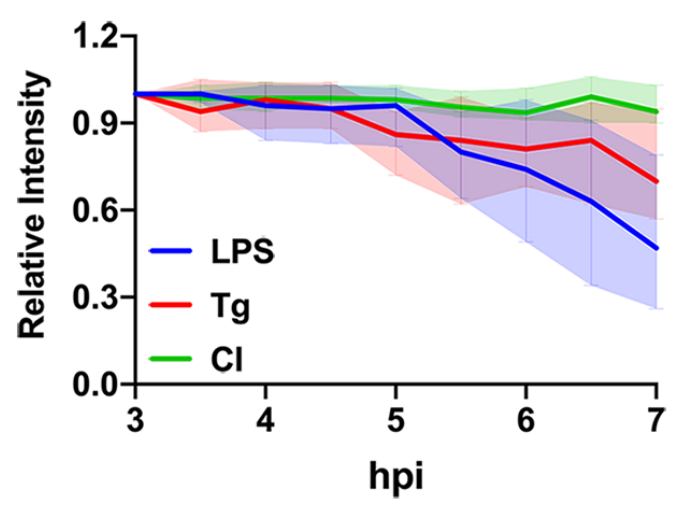

(C)

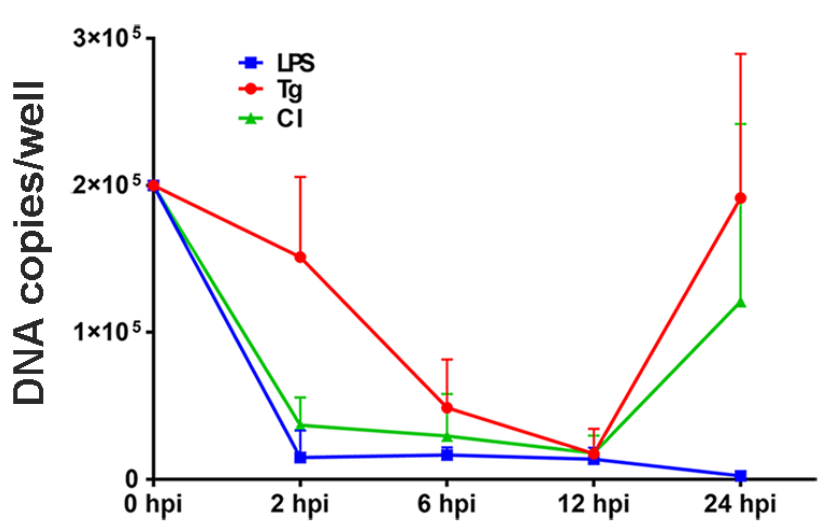

(B)

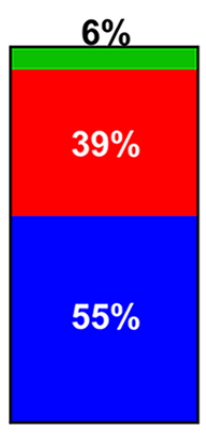

$\mathrm{Tg}$

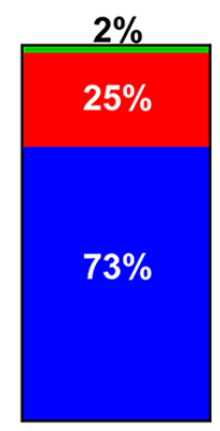

CI
T. gondii - positive MMs
$3 \%$

$18 \%$

$79 \%$

LPS

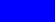

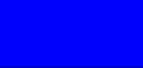

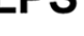

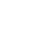

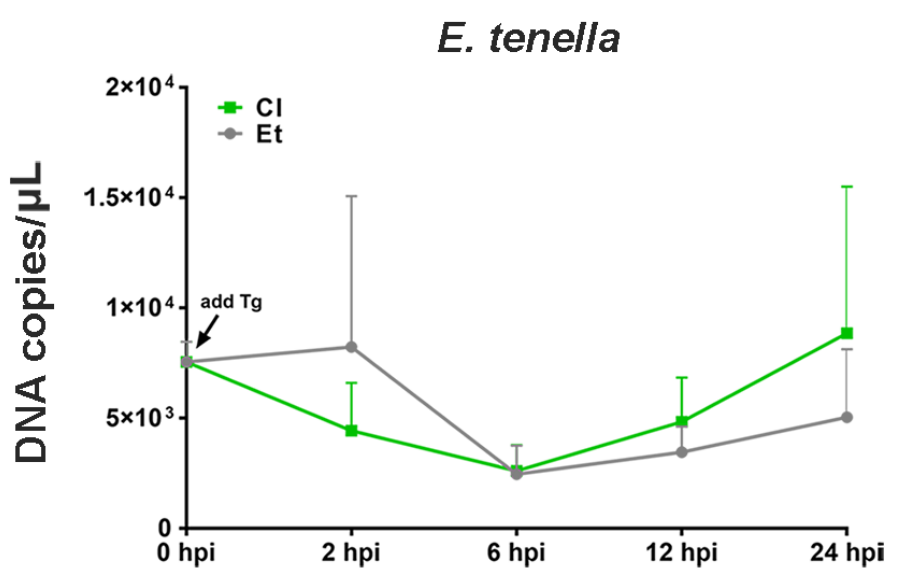

\section{Figure 3}

Quantitative analysis of T. gondii (A) Assessment of intracellular survival of T. gondii ( $n=27-30$ ) by measurement of Relative Intensity (RI) at 3-7 hpi. RI = initial mean intensity of fluorescence for each parasite / mean intensity of fluorescence for each parasite per time point. RI = 0 means no signal of fluorescence is detected. Error bar: standard error of the mean. (B) T. gondii negative/positive MMs. 100 vital T. gondii-positive MMs in group $\mathrm{Cl}$ and $\mathrm{Tg}$ and $71 \mathrm{MMs}$ in group LPS (due to low numbers of surviving parasites) were selected and marked randomly, i.e. 1-2 vital intracellular tachyzoites / cell were captured at $7 \mathrm{hpi}$ and again at $20 \mathrm{hpi}$. The rate of T. gondii-negative/positive cells was calculated. Blue column, MMs with reduced numbers of or non-vital T. gondii; Red column, MMs with constant numbers of vital T. gondii; Green column, MMs with replicating T. gondii. (C) Parasite quantities (DNA copies) during sequential co-infection. Parasite replication is represented as mean value with standard deviation $(n=3-5$ per time point). LPS: LPS-treatment, T. gondii mono-infection; Tg: T. gondii mono-infection; Et: E. tenella mono-infection; Cl: co-infection.

\section{Supplementary Files}


This is a list of supplementary files associated with this preprint. Click to download.

- Graphicalabstractimage920.jpg

- Additionalfile1.mp4 